\title{
Some Recurrence Relations and Hilbert Series of Right-Angled Affine Artin Monoid $M\left(\widetilde{D}_{n}^{\infty}\right)$
}

\author{
Young Chel Kuwn ${ }^{1},{ }^{1}$ Zaffar Iqbal, ${ }^{2}$ Abdul Rauf Nizami, ${ }^{3}$ \\ Mobeen Munir $\left(\mathbb{D},{ }^{4}\right.$ Sana Riaz, ${ }^{2}$ and Shin Min Kang $\mathbb{D}^{5,6}$ \\ ${ }^{1}$ Department of Mathematics, Dong-A University, Busan 49315, Republic of Korea \\ ${ }^{2}$ Department of Mathematics, University of Gujrat, Gujrat, Pakistan \\ ${ }^{3}$ Faculty of Information Technology, University of Central Punjab, Lahore, Pakistan \\ ${ }^{4}$ University of Education, Township Lahore, Pakistan \\ ${ }^{5}$ Department of Mathematics and Research Institute of Natural Science, Gyeongsang National University, \\ Jinju 52828, Republic of Korea \\ ${ }^{6}$ Center for General Education, China Medical University, Taichung 40402, Taiwan \\ Correspondence should be addressed to Mobeen Munir; mmunir@ue.edu.pk and Shin Min Kang; smkang@gnu.ac.kr
}

Received 14 March 2018; Accepted 21 June 2018; Published 17 July 2018

Academic Editor: Raúl E. Curto

Copyright (C) 2018 Young Chel Kuwn et al. This is an open access article distributed under the Creative Commons Attribution License, which permits unrestricted use, distribution, and reproduction in any medium, provided the original work is properly cited.

We find the Hilbert series of the right-angled affine Artin monoid $M\left(\widetilde{D}_{n}^{\infty}\right)$. We also discuss its recurrence relation and the growth rate.

\section{Introduction}

Coxeter groups were introduced by Coxeter in 1934 as abstract form of reflection groups. These groups were classified into two categories in 1935 also by Coxeter: spherical and affine. In the list of spherical Coxeter groups, $A_{n}$ is the first. The Artin group associated with $A_{n}$ is the braid group. Cardinality is an invariant of graded algebraic structures. Hilbert series deals with the cardinality of elements in the graded algebraic structures. In [1] Iqbal gave a linear system for the reducible and irreducible words of the braid monoid $M B_{n}$, which leads to compute the Hilbert series of $M B_{n}$. In [2] Iqbal and Yousaf computed the Hilbert series of the braid monoid $\mathrm{MB}_{4}$ in band generators. In [3] Berceanu and Iqbal proved that the growth rate of all the spherical Artin monoids is less than 4. In [4] Iqbal et al. studied the braid monoid $M\left(\widetilde{A}_{n}^{\infty}\right)$ of the affine type $\widetilde{A}_{n}$ of the Coxeter systems. Authors also found the recurrence relations, the growth series of $M\left(\widetilde{A}_{n}^{\infty}\right)$, and proved that the growth rate of $M\left(\widetilde{A}_{n}^{\infty}\right)$ is unbounded (see Figure 1).
In the present paper we study the affine-type Coxeter group $\widetilde{D}_{n}$ and find the Hilbert series (or spherical growth series) of the associated right-angled affine Artin monoid $M\left(\widetilde{D}_{n}^{\infty}\right)$. We also discuss its recurrence relations and the growth rate. For detailed explanation of related concepts and basic ideas about Coxeter groups and its types, readers are referred to [4] and references therein.

The affine (or infinite) Coxeter groups form another important series of Coxeter groups. These well-known affine Coxeter groups are $\widetilde{A}_{n}, \widetilde{B}_{n}, \widetilde{C}_{n}, \widetilde{D}_{n}, \widetilde{E}_{6}, \widetilde{E}_{7}, \widetilde{E}_{8}, \widetilde{G}_{2}$, and $\widetilde{I}_{1}$ (for details, see [5]). In [3] authors proved that the universal upper bound for all the spherical Artin monoids is less than 4.

In this work we discuss right-angled affine Artin monoids; specifically, we study the affine monoid $M\left(\widetilde{D}_{n}^{\infty}\right)$ and compute its Hilbert series. We show that the growth of $M\left(\widetilde{D}_{n}^{\infty}\right)$ series is bounded above by 4 . Along with Hilbert series we also compute the recurrence relations related to $M\left(\widetilde{D}_{n}^{\infty}\right)$. We give a conjecture about the growth rate of 


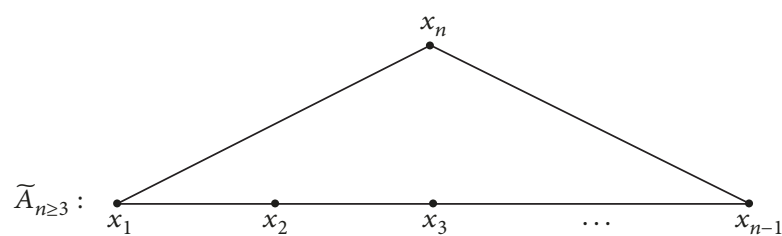

Figure 1

$\left(\widetilde{D}_{n}\right)_{n \geq 5}$

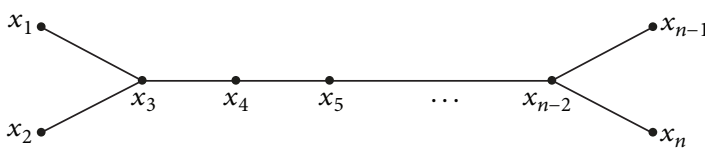

FIgURE 2

$M\left(\widetilde{D}_{n}^{\infty}\right)$, and the growth rates (maximal roots of the characteristic polynomial $\left.D_{n}(\lambda)\right)$ are computed using the softwares Drive6 and Mathematica.

The monoid $M\left(\widetilde{D}_{n}^{\infty}\right)$ is represented by its Coxeter graph as shown in Figure 2.

Here $x_{1}, x_{2}, \ldots, x_{n}$ are vertices of the graph and all the labels are $\infty$. If all the labels in a Coxeter diagram are replaced by $\infty$, then there is no relation between the adjacent edges. Hence we have the associated right-angled Artin groups and the associated right-angled Artin monoids denoted by $G\left(\widetilde{D}_{n}^{\infty}\right)$ and $M\left(\widetilde{D}_{n}^{\infty}\right)$, respectively.

In a monoid the relation $\alpha=\beta$ will be written as $\alpha>\beta$ in the length-lexicographic order. Let $\alpha_{1}=u w$ and $\alpha_{2}=w v$; then the word of the form $u w v$ is said to be an ambiguity. If $\alpha_{1} v=u \alpha_{2}$ is in the length-lexicographic order, then we say that the ambiguity $u w v$ is solvable. Such a presentation is complete if and only if all the ambiguities are solvable. Corresponding to the relation $\alpha=\beta$, the changes $\gamma \alpha \delta \longrightarrow$ $\gamma \beta \delta$ give a rewriting system. A complete presentation is equivalent to a confluent rewriting system. In a complete presentation of a monoid, word containing $\alpha$ will be called reducible word and a word that does not contain $\alpha$ will be called an irreducible word or canonical word. In a presentation of a monoid we fix a total order $x_{1}<x_{2}<\cdots<x_{n}$ on the generators. Hence clearly we have the following.

Lemma 1. The monoid $M\left(\widetilde{D}_{n}^{\infty}\right)$ has the following presentation:

$$
\begin{gathered}
M\left(\widetilde{D}_{n}^{\infty}\right)=\left\langle\begin{array}{c}
x_{1}, x_{2}, \ldots, x_{n} \mid \\
x_{2} x_{1}=x_{1} x_{2}, \\
x_{j} x_{i}=x_{i} x_{j}, 3 \leq i+2 \leq j \leq n-1, j \neq 3 \\
x_{n} x_{k}=x_{k} x_{n}, k \neq n-2
\end{array}\right\rangle .
\end{gathered}
$$

\section{Recurrence Relations of the Monoid $M\left(\widetilde{D}_{n}^{\infty}\right)$}

In this section we discuss few interesting results relating to the recurrence relations of $M\left(\widetilde{D}_{n}^{\infty}\right)$. First we talk about the solution of the system of linear recurrences.
Consider a system [6] of linear recurrences

$$
\begin{aligned}
& u_{1}(t+1)=a_{11}(t) u_{1}(t)+\cdots+a_{1 n}(t) u_{n}(t)+f_{1}(t) \\
& u_{2}(t+1)=a_{11}(t) u_{1}(t)+\cdots+a_{1 n}(t) u_{n}(t)+f_{2}(t)
\end{aligned}
$$

$$
u_{n}(t+1)=a_{11}(t) u_{1}(t)+\cdots+a_{1 n}(t) u_{n}(t)+f_{n}(t) .
$$

This system can be written as $u(t+1)=A(t) u(t)+f(t)$, where

$$
\begin{aligned}
& u(t)=\left[\begin{array}{c}
u_{1}(t) \\
\vdots \\
u_{n}(t)
\end{array}\right], \\
& A(t)=\left[\begin{array}{ccc}
a_{11}(t) & \cdots & a_{1 n}(t) \\
\vdots & \ddots & \vdots \\
a_{n 1}(t) & \cdots & a_{n n}(t)
\end{array}\right], \\
& f(t)=\left[\begin{array}{c}
f_{1}(t) \\
\vdots \\
f_{n}(t)
\end{array}\right] .
\end{aligned}
$$

The solution (which we need in our work) of the homogenous equation $u(t+1)=A(t) u(t)$ is given by $u(t)=c_{1} \lambda_{1}^{t} u^{1}+$ $\cdots+c_{k} \lambda_{k}^{t} u^{k}$, where $\lambda_{1}, \ldots, \lambda_{k}$ are the eigenvalues of $A(t)$ and $u^{i}$ is an eigenvector corresponding to $\lambda_{i}$. The largest eigenvalue is the growth rate of the sequence $\left(u_{i}(t)\right)$ (by the definition of growth rate).

Let $c_{k}=\#\{$ canonical words of length $k\}$ and $c_{k ; i}=$ $\#\left\{\right.$ canonical words starting with $x_{i}$ of length $\left.k\right\}$. Then we have the following.

Lemma 2. The monoid $M\left(\widetilde{D}_{n}^{\infty}\right)$ satisfies the following recurrence relations:

(a) $c_{0}=1, c_{1 ; i}=1$, and $c_{k}=\sum_{i=1}^{n} c_{k ; i},(k \geq 1)$.

(b) $c_{k ; i}(k \geq 1)$ are given by the recurrence

$$
c_{k ; i}= \begin{cases}\sum_{j=i}^{n} c_{k-1 ; j}, & i=1,2, \\ c_{k ; 1}, & i=3, \\ \sum_{j=i-1}^{n} c_{k-1 ; j}, & i=4,5, \ldots, n-1, \\ c_{k-1 ; n-2}+c_{k-1 ; n}, & i=n .\end{cases}
$$

Let $K_{n}$ be a Coxeter diagram $\left(K_{n}\right.$ is similar to the standard Coxeter diagram $D_{n}$ but opposite in direction) (see Figure 3 ).

We use $M\left(K_{n}^{\infty}\right)$ in the solution of the recurrence of $M\left(\widetilde{D}_{n}^{\infty}\right)$. Therefore we have the following.

Lemma 3. The monoid $M\left(K_{n}^{\infty}\right)$ satisfies the recurrence relations 


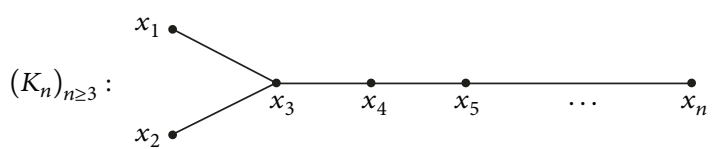

Figure 3

(a) $c_{0}=1, c_{1 ; i}=1$, and $c_{k}=\sum_{i=1}^{n} c_{k ; i},(k \geq 1)$.

(b) $c_{k ; i}(k \geq 1)$ are given by the recurrence

$$
c_{k ; i}= \begin{cases}\sum_{j=i}^{n} c_{k-1 ; j}, & i=1,2, \\ c_{k ; 1}, & i=3, \\ \sum_{j=i-1}^{n} c_{k-1 ; j}, & i=4,5, \ldots, n\end{cases}
$$

Let $K_{n}(\lambda)$ and $D_{n}(\lambda)$ denote the characteristic polynomials of the system of recurrence relations of the monoids $M\left(K_{n}^{\infty}\right)$ and $M\left(\widetilde{D}_{n}^{\infty}\right)$, respectively. Then we have the following.

Lemma 4. The polynomials $\left(K_{n}(\lambda)\right)_{n \geq 3}$ satisfy the recurrence relation

$$
K_{n}(\lambda)=\lambda K_{n-1}(\lambda)-\lambda K_{n-2}(\lambda), \quad n \geq 3
$$

with the initial values $K_{3}(\lambda)=\lambda^{3}-3 \lambda^{2}+\lambda$ and $K_{4}(\lambda)=$ $\lambda^{4}-4 \lambda^{3}+3 \lambda^{2}-\lambda$.

Proof. Let $P_{n}$ be the matrix of order $n \times n$ of the recurrences given in the Lemma 3 . Then the characteristic polynomial $K_{n}(\lambda)$ of $P_{n}$ is

$$
K_{n}(\lambda)=\left|\begin{array}{ccccccc}
\lambda-1 & -1 & \cdots & -1 & -1 & -1 & -1 \\
0 & \lambda-1 & \cdots & -1 & -1 & -1 & -1 \\
-1 & -1 & \cdots & -1 & -1 & -1 & -1 \\
\vdots & \vdots & & \vdots & \vdots & \vdots & \vdots \\
0 & 0 & \cdots & 0 & -1 & \lambda-1 & -1 \\
0 & 0 & \cdots & 0 & 0 & -1 & \lambda-1
\end{array}\right| .
$$

We write $K_{n}(\lambda)=S_{n}(\lambda)+T_{n}(\lambda)$, where the determinants $S_{n}(\lambda)$ and $T_{n}(\lambda)$ are obtained by splitting $K_{n}(\lambda)$ such that the last rows of $S_{n}(\lambda)$ and $T_{n}(\lambda)$ are $(0, \ldots, 0, \lambda)$ and $(0, \ldots, 0,-1,-1)$, respectively. Therefore easily we have $S_{n}(\lambda)=\lambda K_{n-1}(\lambda)$ and

$$
T_{n}(\lambda)=\left|\begin{array}{ccccccc}
\lambda-1 & -1 & \cdots & -1 & -1 & -1 & -1 \\
0 & \lambda-1 & \cdots & -1 & -1 & -1 & -1 \\
-1 & -1 & \cdots & -1 & -1 & -1 & -1 \\
\vdots & \vdots & & \vdots & \vdots & \vdots & \vdots \\
0 & 0 & \cdots & 0 & -1 & \lambda-1 & -1 \\
0 & 0 & \cdots & 0 & 0 & -1 & -1
\end{array}\right| .
$$

Subtracting last column from 2 nd last column of $T_{n}(\lambda)$ and after few easy computations we have the recurrence relation $K_{n}(\lambda)=\lambda K_{n-1}(\lambda)-\lambda K_{n-2}(\lambda), n \geq 3$.
Here we have an explicit formula to compute $K_{n}(\lambda)$.

Lemma 5. Let $q=\sqrt{\lambda^{2}-4 \lambda}$; then we have the following:

(1) $K_{2 m}(\lambda)=\left(1 / 2^{2 m}\right) \sum_{k=0}^{2 m}\left(\begin{array}{c}2 m \\ k\end{array}\right) t^{2 m-k} q^{2 k}(a+b)$,

(2) $K_{2 m+1}(\lambda)=\left(1 / 2^{2 m+1}\right) \sum_{k=0}^{2 m+1}\left(\begin{array}{c}2 m+1 \\ k\end{array}\right) t^{2 m-k+1} q^{2 k+1}(a-$ b), where

$$
\begin{aligned}
& a=\frac{4 t\left(q\left(t^{3}-3 t+1\right)+\left(t^{3}-5 t^{2}+5 t-2\right)\right)}{q(t+q)^{3}}, \\
& b=\frac{4 t\left(q\left(t^{3}-3 t+1\right)-\left(t^{3}-5 t^{2}+5 t-2\right)\right)}{q(t-q)^{3}} .
\end{aligned}
$$

Proof. Let $K_{n}(\lambda)=\alpha^{n}$. Then $K_{n}(\lambda)=\lambda K_{n-1}(\lambda)-\lambda K_{n-2}(\lambda)$ can be written as $\alpha^{n}=\lambda \alpha^{n-1}-\lambda \alpha^{n-2}$; i.e., $\alpha^{2}-\lambda \alpha+\lambda=0$. This characteristic equation has the roots $\alpha=\left(\lambda \pm \sqrt{\lambda^{2}-4 \lambda}\right) / 2$. Let $\alpha_{1}=\left(\lambda+\sqrt{\lambda^{2}-4 \lambda}\right) / 2$ and $\alpha_{2}=\left(\lambda-\sqrt{\lambda^{2}-4 \lambda}\right) / 2$. Then we have the solution of the recurrence given by $K_{n}(\lambda)=a \alpha_{1}^{n}+$ $b \alpha_{2}^{n}$, where $a$ and $b$ are constants to determine. Since $K_{3}(\lambda)=$ $\lambda^{3}-3 \lambda^{2}+\lambda$ and $K_{4}(\lambda)=\lambda^{4}-4 \lambda^{3}+3 \lambda^{2}-\lambda$, therefore

$$
\begin{array}{r}
\lambda^{3}-3 \lambda^{2}+\lambda=\alpha_{1}^{3} a+\alpha_{2}^{3} b, \\
\lambda^{4}-4 \lambda^{3}+3 \lambda^{2}-\lambda=\alpha_{1}^{4} a+\alpha_{2}^{4} b .
\end{array}
$$

Solving these equations we get $a=\left(4 t / q(t+q)^{3}\right)\left(q\left(t^{3}-3 t+\right.\right.$ $\left.1)+\left(t^{3}-5 t^{2}+5 t-2\right)\right)$ and $b=\left(4 t / q(t-q)^{3}\right)\left(q\left(t^{3}-3 t+1\right)-\right.$ $\left.\left(t^{3}-5 t^{2}+5 t-2\right)\right)$. Hence

$$
\begin{aligned}
K_{n}(\lambda)= & \frac{a(t+q)^{n}+b(t-q)^{n}}{2^{n}} \\
= & \frac{a}{2^{n}} \sum_{k=0}^{n}\left(\begin{array}{l}
n \\
k
\end{array}\right) t^{n-k} q^{k} \\
& +\frac{b}{2^{n}} \sum_{k=0}^{n}\left(\begin{array}{l}
n \\
k
\end{array}\right) t^{n-k}(-q)^{k} .
\end{aligned}
$$

For even and odd values of $n$ we have

$$
\begin{aligned}
& K_{2 m}(\lambda)=\frac{1}{2^{2 m}} \sum_{k=0}^{2 m}\left(\begin{array}{c}
2 m \\
k
\end{array}\right) t^{2 m-k} q^{2 k}(a+b), \\
& K_{2 m+1}(\lambda) \\
& =\frac{1}{2^{2 m+1}} \sum_{k=0}^{2 m+1}\left(\begin{array}{c}
2 m+1 \\
k
\end{array}\right) t^{2 m-k+1} q^{2 k+1}(a-b) .
\end{aligned}
$$

Theorem 6. The polynomials $\left(D_{n}(\lambda)\right)_{n \geq 5}$ satisfy the recurrence relation

$$
D_{n}(\lambda)=(\lambda-1) K_{n-1}(\lambda)-\lambda^{2} K_{n-4}(\lambda), \quad n \geq 5
$$


with $D_{0}(\lambda)=1, D_{1}(\lambda)=\lambda-1, D_{2}(\lambda)=(\lambda-1)^{2}, D_{3}(\lambda)=$ $\lambda\left(\lambda^{2}-3 \lambda+1\right)$, and $D_{4}(\lambda)=\lambda^{2}\left(\lambda^{2}-4 \lambda+3\right)$ being the initial values.

Proof. Let $M_{n}$ be the matrix of order $n \times n$ of the recurrences given in Lemma 2. Then the characteristic polynomial of $M_{n}$ is

$$
D_{n}(\lambda)=\left|\begin{array}{ccccccc}
\lambda-1 & -1 & \cdots & -1 & -1 & -1 & -1 \\
0 & \lambda-1 & \cdots & -1 & -1 & -1 & -1 \\
-1 & -1 & \cdots & -1 & -1 & -1 & -1 \\
\vdots & \vdots & & \vdots & \vdots & \vdots & \vdots \\
0 & 0 & \cdots & -1 & \lambda-1 & -1 & -1 \\
0 & 0 & \cdots & 0 & -1 & \lambda-1 & -1 \\
0 & 0 & \cdots & 0 & -1 & 0 & \lambda-1
\end{array}\right| .
$$

Write $D_{n}(\lambda)=U_{n}(\lambda)+V_{n}(\lambda)$, where the determinants $U_{n}(\lambda)$ and $V_{n}(\lambda)$ are obtained by splitting $D_{n}(\lambda)$ such that the last row of $U_{n}(\lambda)$ is $(0, \ldots, 0, \lambda-1)$ and the last row of $V_{n}(\lambda)$ is $(0, \ldots, 0,-1,0,0)$. Hence easily we have $U_{n}(\lambda)=(\lambda-$ 1) $K_{n-1}(\lambda)$. Subtracting last column from 3 rd last and 2 nd last columns of $V_{n}(\lambda)$, respectively, we have $V_{n}(\lambda)=-\lambda^{2} K_{n-4}(\lambda)$. Hence

$$
D_{n}(\lambda)=(\lambda-1) K_{n-1}(\lambda)-\lambda^{2} K_{n-4}(\lambda), \quad n \geq 5 .
$$

\section{The Hilbert Series of the Monoid $M\left(\widetilde{D}_{n}^{\infty}\right)$}

Now we compute the Hilbert series of $M\left(\widetilde{D}_{n}^{\infty}\right)$. For this we need to fix some notations first. Let $\mathscr{H}_{M}^{(n)}(t)=\sum_{k \geq 0} c_{k} t^{k}$ denote the Hilbert series of $M\left(\widetilde{D}_{n}^{\infty}\right)$, where $c_{k}=\#$ \{canonical words of length $k\}$, and $\mathscr{H}_{M ; i}^{(n)}(t)=\sum_{k \geq 0} c_{k ; i}^{(n)} t^{k}$ denote the Hilbert series of $M\left(\widetilde{D}_{n}^{\infty}\right)$ of words starting with $x_{i}$, where $c_{k ; i}=\#\left\{\right.$ canonical words starting with $x_{i}$ of length $\left.k\right\}$.

Theorem 7. The Hilbert series of $M\left(\widetilde{D}_{n}^{\infty}\right)$ is given by the following:
(1) $\mathscr{H}_{M}^{(n)}(t)=1+\sum_{i=1}^{n} \mathscr{H}_{M ; i}^{n}(t)$,
(2) $\mathscr{H}_{M ; i}^{(n)}(t)=t+t \sum_{j=i}^{n} \mathscr{H}_{M ; j}^{(n)}(t), i=1,2$,
(3) $\mathscr{H}_{M ; 1}^{(n)}(t)=\mathscr{H}_{M ; 3}^{(n)}(t)$,
(4) $\mathscr{H}_{M ; i}^{(n)}(t)=t+t \sum_{j=i-1}^{n} \mathscr{H}_{M ; j}^{(n)}(t), i=4,5, \ldots, n-1$,
(5) $\mathscr{H}_{M ; n}^{(n)}(t)=t+t \mathscr{H}_{M ; n-2}^{(n)}(t)+t \mathscr{H}_{M ; n}^{(n)}(t)$.

Proof. (1) From Lemma 2 we have $c_{k}=\sum_{i=1}^{n} c_{k ; i},(k \geq 1)$. Therefore

$$
\begin{aligned}
\mathscr{H}_{M}^{n}(t) & =\sum_{k \geq 0} c_{k} t^{k}=c_{0}+\sum_{k \geq 1} c_{k} t^{k}=1+\sum_{k \geq 1} \sum_{i=1}^{n} c_{k ; i} t^{k} \\
& =1+\sum_{i=1}^{n} \sum_{k \geq 1} c_{k ; i} t^{k}=1+\sum_{i=1}^{n} \mathscr{H}_{M ; i}^{n}(t)
\end{aligned}
$$

(2) From Lemma 2 we have $c_{k ; i}=\sum_{j=i}^{n} c_{k-1 ; j}(i=1,2)$. Hence

$$
\begin{aligned}
\mathscr{H}_{M ; i}^{(n)}(t) & =\sum_{k \geq 1}^{n} c_{k ; i}(t) t^{k}=c_{1 ; i}(t) t+\sum_{k \geq 2}^{n} c_{k ; i}(t) t^{k} \\
& =t+\sum_{k \geq 2}^{n} \sum_{j=i}^{n} c_{k-1 ; j}(t) t^{k} \\
& =t+t \sum_{j=i}^{n} \sum_{k \geq 2}^{n} c_{k-1 ; j}(t) t^{k-1} \\
& =t+t \sum_{j=i}^{n} \mathscr{H}_{M ; i}^{(n)}(t) .
\end{aligned}
$$

Similarly we can easily prove (3), (4), and (5).

The system of equations in Theorem 7 can be written in matrix form as $W_{n} X=B$, where

$$
W_{n}=\left[\begin{array}{ccccccc}
1-t & -t & \cdots & -t & -t & -t & -t \\
0 & 1-t & \cdots & -t & -t & -t & -t \\
-t & -t & \cdots & -t & -t & -t & -t \\
\vdots & \vdots & & \vdots & \vdots & \vdots & \vdots \\
0 & 0 & \cdots & -t & 1-t & -t & -t \\
0 & 0 & \cdots & 0 & -t & 1-t & -t \\
0 & 0 & \cdots & 0 & -t & 0 & 1-t
\end{array}\right],
$$

$$
X=\left[\begin{array}{c}
\mathscr{H}_{M ; 1}^{(n)}(t) \\
\mathscr{H}_{M ; 2}^{(n)}(t) \\
\mathscr{H}_{M ; 3}^{(n)}(t) \\
\vdots \\
\mathscr{H}_{M ; n}^{(n)}(t)
\end{array}\right],
$$

$$
B=\left[\begin{array}{c}
t \\
t \\
t \\
\vdots \\
t
\end{array}\right] .
$$

Lemma 8. In the monoid $M\left(\widetilde{D}_{n}^{\infty}\right)$

$$
\operatorname{det}\left(W_{n}\right)=t^{n} D_{n}\left(\frac{1}{t}\right) .
$$

Proof. The result follows immediately by factoring out $t$ from each row of $\operatorname{det}\left(W_{n}\right)$. 
Lemma 9. In $M\left(\widetilde{D}_{n}^{\infty}\right)$

$$
\begin{aligned}
& \mathscr{H}_{M ; m}^{(n)}(t) \\
& = \begin{cases}\frac{t^{m-1} K_{m-2}(1 / t)}{t^{n} D_{n}(1 / t)}, & 5 \leq m \leq n-1 \\
\frac{(1-t) t^{m-2} K_{m-3}(1 / t)}{t^{n} D_{n}(1 / t)}, & m=n .\end{cases}
\end{aligned}
$$

Proof. The system given in Theorem 7 of $n$ equations in $n$ variables $\mathscr{H}_{M ; i}^{(n)}(t), 1 \leq i \leq n$ is already written in the form $W_{n} X=B$, where $X=\left[\mathscr{H}_{M ; 1}^{(n)}(t), \ldots, \mathscr{H}_{M ; n}^{(n)}(t)\right]^{t}, \operatorname{det}\left(W_{n}\right)=$ $t^{n} D_{n}(1 / t)$, and $B=[t, \ldots, t]^{t}$. Here we have only two cases.

Case I $(5 \leq m \leq n-1)$. By using Cramer's rule we have

$$
\mathscr{H}_{M ; m}^{(n)}(t)=\frac{T_{m}}{\operatorname{det}\left(W_{n}\right)}
$$

where $T_{m}$ is a determinant obtained by replacing $m$ th column of $W_{n}$ by column of $B$; i.e.,

$$
\begin{aligned}
& \mathscr{H}_{M ; m}^{(n)}(t) \\
& =\frac{1}{t^{n} D_{n}(1 / t)}\left|\begin{array}{cccccccc}
1-t & -t & \cdots & -t & t & \cdots & -t & -t \\
0 & 1-t & \cdots & -t & t & \cdots & -t & -t \\
-t & -t & \cdots & -t & t & \cdots & -t & -t \\
\vdots & \vdots & & \vdots & \vdots & & \vdots & \vdots \\
0 & 0 & \cdots & 0 & t & \cdots & 0 & 1-t
\end{array}\right| .
\end{aligned}
$$

Let $C_{i}$ denote the $i$ th column of $T_{m}$. Adding $C_{m}$ in $C_{m+1}, C_{m+2}, \ldots, C_{n}$ of $T_{m}$ and simplifying it we have a determinant of order $m$. Hence

$$
\mathscr{H}_{M ; m}^{(n)}(t)=\frac{1}{t^{n} D_{n}(1 / t)}\left|\begin{array}{ccccc}
1-t & -t & \cdots & -t & t \\
0 & 1-t & \cdots & -t & t \\
-t & -t & \cdots & -t & t \\
\vdots & \vdots & & \vdots & \vdots \\
0 & 0 & \cdots & -t & t
\end{array}\right| .
$$

Now by adding $(m-1)$ th column of the last determinant in its $m$ th column and simplifying it we finally have

$$
\mathscr{H}_{M ; m}^{(n)}(t)=\frac{t^{m-1} K_{m-2}(1 / t)}{t^{n} D_{n}(1 / t)} .
$$

Case II $(m=n)$. Using again Cramer's rule, we have

$$
\begin{aligned}
& \mathscr{H}_{M ; n}^{(n)}(t) \\
& =\frac{1}{t^{n} D_{n}(1 / t)}\left|\begin{array}{ccccccc}
1-t & -t & \cdots & -t & -t & -t & t \\
0 & 1-t & \cdots & -t & -t & -t & t \\
-t & -t & \cdots & -t & -t & -t & t \\
\vdots & \vdots & & \vdots & \vdots & \vdots & \vdots \\
0 & 0 & \cdots & -t & 1-t & -t & t \\
0 & 0 & \cdots & 0 & -t & 1-t & t \\
0 & 0 & \cdots & 0 & -t & 0 & t
\end{array}\right| .
\end{aligned}
$$

Adding $C_{n}$ in $C_{n-2}$ and simplifying we have the result

$$
\mathscr{H}_{M ; m}^{(n)}(t)=\frac{(1-t) t^{m-2} K_{m-3}(1 / t)}{t^{n} D_{n}(1 / t)} .
$$

Now we have our main result.

Theorem 10. The Hilbert series of the monoid $M\left(\widetilde{D}_{n}^{\infty}\right)$ is

$$
\mathscr{H}_{M}^{(n)}(t)=\frac{1}{t^{n} D_{n}(1 / t)}
$$

Proof. From Theorem 7 we have

$$
\begin{aligned}
\mathscr{H}_{M}^{(n)}(t)=1+\mathscr{H}_{M ; 1}^{(n)}(t)+\mathscr{H}_{M ; 2}^{(n)}(t)+\cdots \\
+\mathscr{H}_{M ; n-1}^{(n)}(t)+\mathscr{H}_{M ; n}^{(n)}(t)=\frac{1}{t^{n} D_{n}(1 / t)}\left(t^{n} D_{n}\left(\frac{1}{t}\right)\right. \\
+t^{3}-3 t^{2}+4 t+t^{4} K_{3}\left(\frac{1}{t}\right)+t^{5} K_{4}\left(\frac{1}{t}\right)+\cdots \\
\left.+t^{n-1} K_{n-2}\left(\frac{1}{t}\right)+(1-t) t^{n-1} K_{n-3}\left(\frac{1}{t}\right)\right) \\
=\frac{1}{t^{n} D_{n}(1 / t)}\left((1-t) t^{n-1} K_{n-1}\left(\frac{1}{t}\right)\right. \\
-t^{n-2} K_{n-2}\left(\frac{1}{t}\right)+t^{3}-3 t^{2}+4 t+t^{4} B_{3}\left(\frac{1}{t}\right)+\cdots \\
\left.+t^{n-1} K_{n-2}\left(\frac{1}{t}\right)+(1-t) t^{n-1} K_{n-3}\left(\frac{1}{t}\right)\right) \\
=\frac{1}{t^{n} D_{n}(1 / t)}\left(t^{4} K_{4}\left(\frac{1}{t}\right)+t^{3}-3 t^{2}+4 t\right) \\
=\frac{1}{t^{n} D_{n}(1 / t)}\left(t^{4}\left(\frac{1}{t^{4}}-\frac{4}{t^{3}}+\frac{3}{t^{2}}-\frac{1}{t}\right)+t^{3}-3 t^{2}\right. \\
+4 t)=\frac{1}{t^{n} D_{n}(1 / t)} .
\end{aligned}
$$




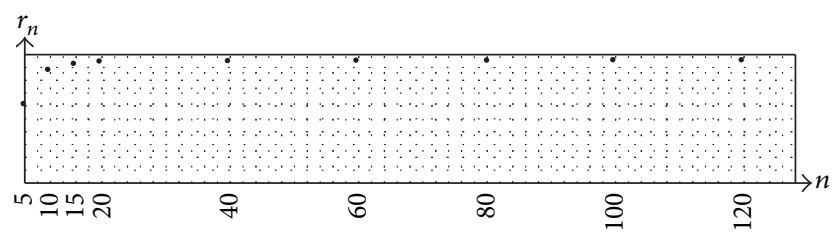

FIgURe 4

\section{Conjecture on the Upper Bound of Growth Rate of $M\left(\widetilde{D}_{n}^{\infty}\right)$}

In this section we compute the growth rates of $M\left(\widetilde{D}_{n}^{\infty}\right)$ and show them on the graph. We see that the growth rate is bounded above by 4 . Let $r_{n}$ be the growth rate (or the maximal root of the polynomial $D_{n}(\lambda)$ ). We compute few initial growth rates (using Mathematica and derive 6) for $M\left(\widetilde{D}_{n}^{\infty}\right)$. Let $r_{n}$ denote the growth rate of $M\left(\widetilde{D}_{n}^{\infty}\right)$; then we have the following few initial values of $r_{n}: r_{5}=3.629, r_{6}=3.732, r_{7}=3.791$, $r_{8}=3.831, r_{9}=3.858, r_{10}=3.879, r_{11}=3.895, r_{12}=3.907$, $r_{13}=3.917, r_{14}=3.926, r_{15}=3.933, r_{16}=3.939, r_{17}=3.944$, $r_{18}=3.948, r_{19}=3.952, r_{20}=3.956$. We also compute $r_{40}=3.984, r_{60}=3.992, r_{80}=3.995, r_{100}=3.996$, and $r_{120}=$ 3.997 (using Mathematica). We have Figure 4 representing the growth rate of $M\left(\widetilde{D}_{n}^{\infty}\right)$.

We observe that the growth rate for $M\left(\widetilde{D}_{n}^{\infty}\right)$ approaching 4 as $n$ approaches $\infty$. Hence at the end we have the following.

Conjecture. The growth rate of $M\left(\widetilde{D}_{n}^{\infty}\right)$ is bounded above by 4.

\section{Data Availability}

No such data has been used to prove these results.

\section{Conflicts of Interest}

The authors declare that they have no conflicts of interest.

\section{Authors' Contributions}

All authors contributed equally to the writing of this paper. All authors read and approved the final manuscript.

\section{Acknowledgments}

This study was supported by research funds from Dong-A University.

\section{References}

[1] Z. Iqbal, "Hilbert series of positive braids," Algebra Colloquium, vol. 18, no. Special Issue 1, pp. 1017-1028, 2011.

[2] Z. Iqbal and S. Yousaf, "Hilbert series of the braid monoid $M B_{4}$ in band generators," Turkish Journal of Mathematics, vol. 38, no. 6, pp. 977-984, 2014.

[3] B. Berceanu and Z. Iqbal, "Universal upper bound for the growth of artin monoids," Communications in Algebra, vol. 43, no. 5, pp. 1967-1982, 2015.
[4] Z. Iqbal, S. Batool, and M. Akram, "Hilbert series of rightangled Affine Artin Monoid $M\left(\widetilde{A}_{n}^{\infty}\right)$," Kuwait Journal of Science, vol. 44, no. 4, pp. 19-27, 2017.

[5] H. S. Coxeter, Regular Complex Polytopes, Cambridge University Press, 2nd edition, 1991.

[6] W. G. Kelley and A. C. Peterson, Difference Equations: An Introduction with Applications, Academic Press, New York, NY, USA, 2nd edition, 2001. 


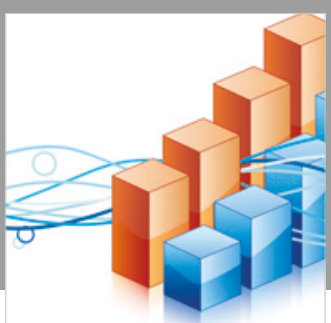

Advances in

Operations Research

\section{-n-m}
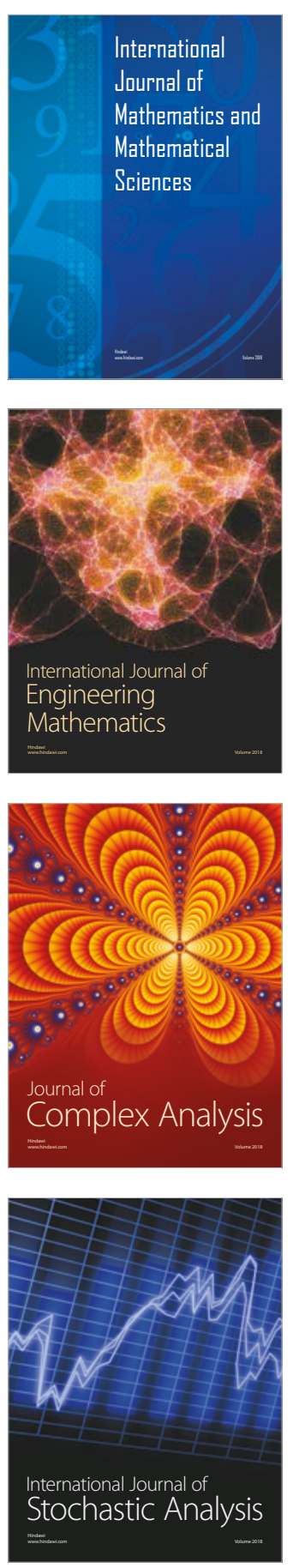
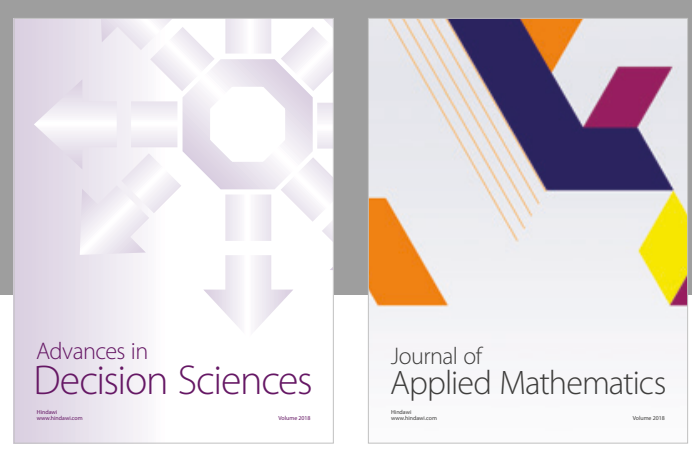

Journal of

Applied Mathematics
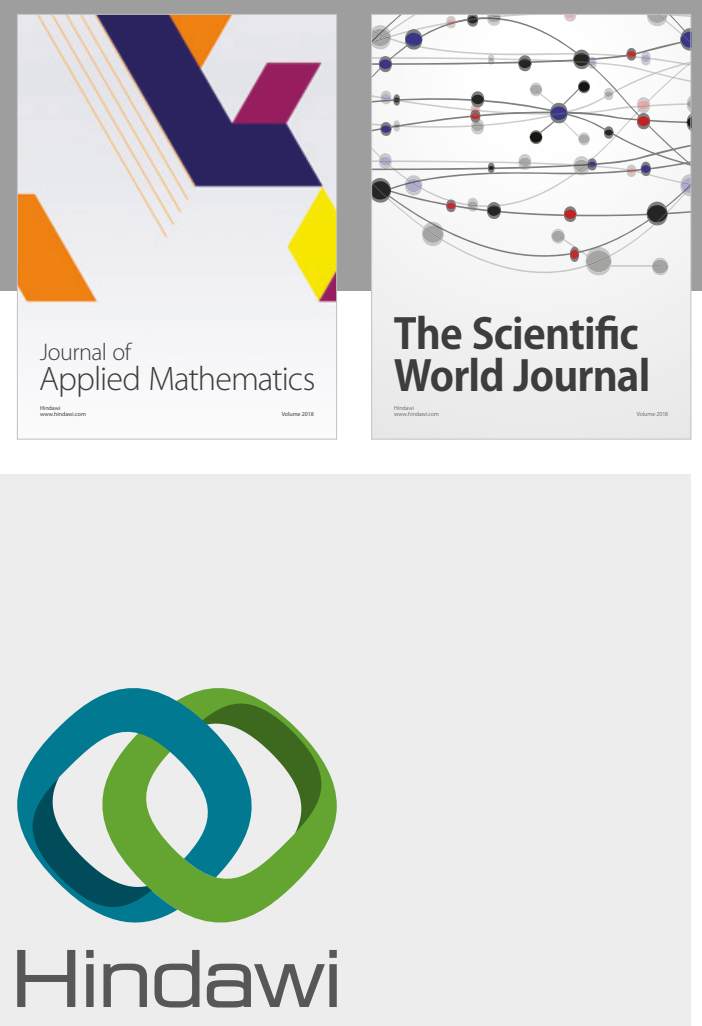

Submit your manuscripts at

www.hindawi.com

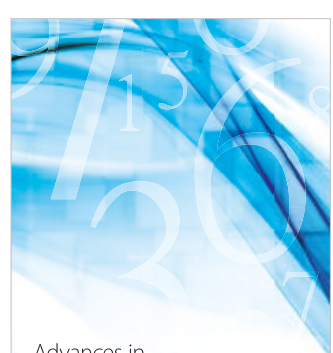

Advances in
Numerical Analysis
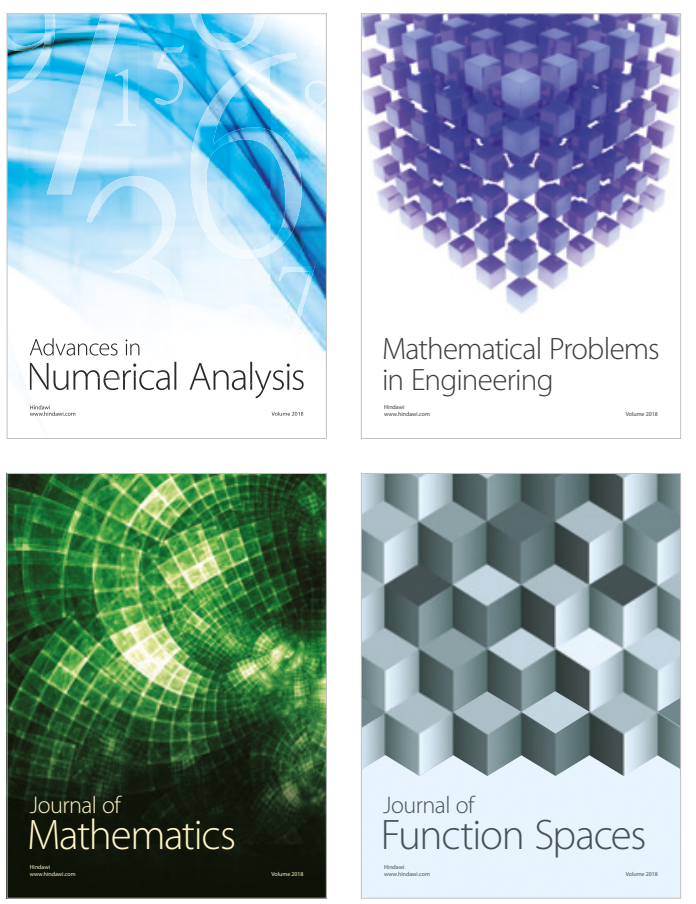

Mathematical Problems in Engineering

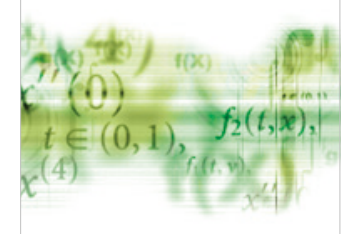

International Journal of

Differential Equations

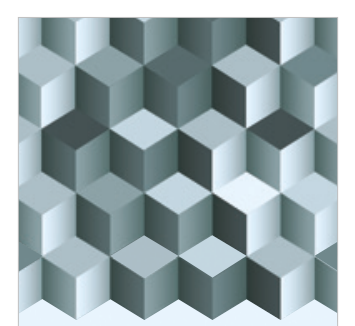

Journal of

Function Spaces

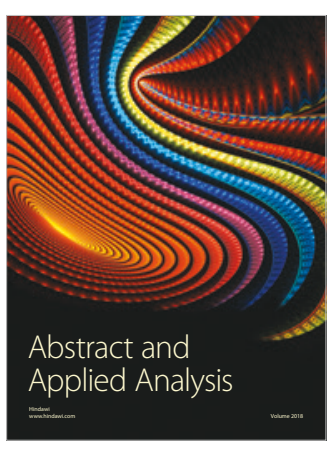

The Scientific

World Journal

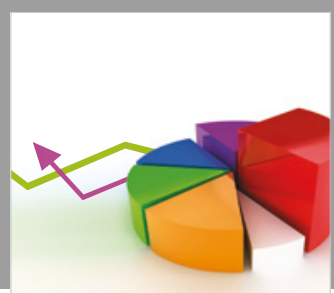

Journal of

Probability and Statistics
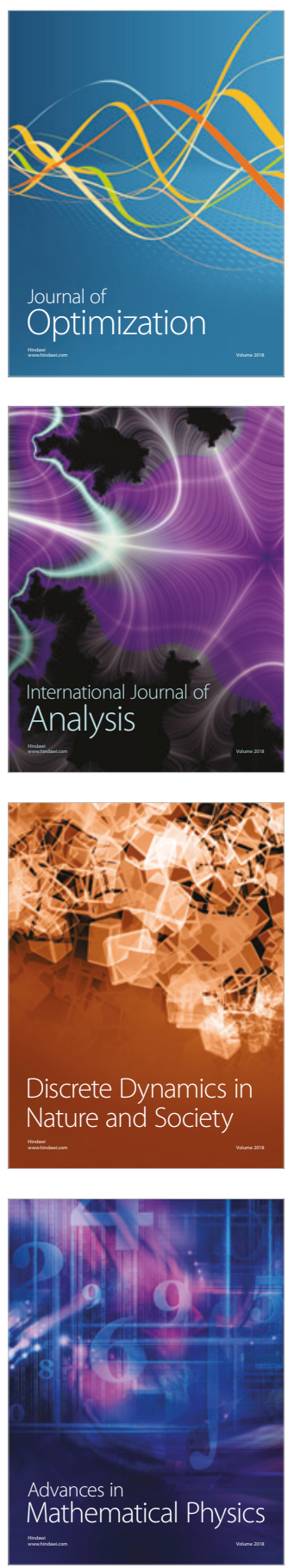